\title{
Ultrasonography plus radiography was as effective as intravenous urography for detecting abnormalities in men with proven urinary tract infection
}

Andrews S, Brooks PT, Hanbury DC, et al. Ultrasonography and abdominal radiography versus intravenous urography in investigation of urinary tract infection in men: prospective incident cohort study. BMJ 2002 Feb;324:454-6.

\section{QUESTION: What is the accuracy of ultrasonography plus abdominal radiography for detecting urinary tract abnormalities in men with proven urinary tract infection (UTI)?}

\section{Design}

Blinded comparison of ultrasonography plus radiography with intravenous urography (IVU) (diagnostic standard).

\section{Setting}

The urology and nephrology departments of a district general hospital in Hertfordshire, England, UK.

\section{Patients}

114 consecutive men (mean age $54 \mathrm{y}$ ), with proven UTI. Complete data were obtained for 100 men.

\section{Description of tests and diagnostic \\ standard}

Ultrasound examination was done with a $3.5-\mathrm{MHz}$ probe. Each kidney was assessed in the longitudinal and tranverse planes, and renal length was recorded. The bladder was assessed full and after micturition. Urinary flow rates were assessed with a Dantec Urodyn 1000. The diagnostic standard, IVU, comprised a full-length plain film, an immediate cross-kidney film after injection of contrast media, a 7-minute cross-kidney film, a 15-minute full-length film, and a bladder film after micturition. Tomography was done at the discretion of the supervising radiologist.

\section{Main outcome measures}

Sensitivity, specificity, and likelihood ratios.

\section{Main results}

53 men had a detectable abnormality that was considered to be clinically significant. The table summarises the test properties of ultrasonography plus radiography. Ultrasonography detected hydronephrosis in 8 patients, 1 of whom was shown to be normal on IVU. Ultrasonography alone missed 5 of 6 patients with urinary tract stones, but all were detected with the addition of radiography. Both ultrasonography plus radiography and IVU found 3 small, scarred kidneys; ultrasonography plus radiography misdiagnosed 1 small, scarred kidney.

Test properties of ultrasonography plus radiography for detecting abnormalities in men with proven urinary tract infection *

\begin{tabular}{|c|c|c|c|}
\hline Sensitivity (95\% CI) & Specificity (Cl) & $+\mathrm{LR}$ & -LR \\
\hline $100 \%$ (94 to 100$)$ & $93 \%$ (81 to 99$)$ & 14.67 & 0 \\
\hline
\end{tabular}

Both methods identified a pelvic kidney. Ultrasonography plus radiography identified 34 patients with a poorly emptying bladder, whereas IVU identified 26.

\section{Conclusion}

Ultrasonography plus radiography was as effective as intravenous urography for detecting clinically important abnormalities in men with proven urinary tract infection.

\section{COMIMENTARY}

UTIs in men are uncommon, and the diagnostic strategies to detect genitourinary abnormalities in these men are variable. The most common initial test is IVU, but a safer and less expensive alternative would be useful. In the study by Andrews et al, about $50 \%$ of the men had no abnormality detected on any of the diagnostic tests. The remaining participants had abnormal findings, such as excessive urine in the bladder after micturition, hydronephrosis, stones, renal scars, or a pelvic kidney. The performance properties of ultrasonography plus radiography are sufficiently close to IVU to make it a reasonable substitution. The authors suggest an algorithm that would have had the following results in this sample after ultrasonography plus radiography: $18 \%$ of patients would have been referred for IVU (abnormal findings in the upper tract), and 8\% would have been referred for unnecessary work-up of lower urinary tract problems. However, this algorithm has not been evaluated in an independent population.

It is unclear which men with UTI need any evaluation, or when it should be done (ie, after the first infection or after subsequent infections). It would have been helpful to stratify the men by the number of previous UTIs and by age because one would expect the age range of 18 to 88 years to include men with very different probabilities of prostate hypertrophy. Referral bias could be a concern because the study was done in a urology and nephrology referral centre.

Local differences in the cost and convenience of testing might make a difference in a cost-benefit analysis, although the risks associated with IVU are greater (about 4 times the ionising radiation and some risk from intravenous contrast). Other tests used in this study (eg, urine flow rate, cystoscopy, and transrectal ultrasonography) could play an important role in diagnosis, but they were not studied in a controlled fashion. The clinical bottom line is that there is no harm in starting with ultrasonography plus radiography when investigating the cause of UTI in men. A negative result can conclude the work-up, and a positive result can lead to further testing or a referral to a urology or nephrology unit.

Thomas Fekete, MD Temple University Hospital Philadelphia, Pennsylvania, USA 